\title{
Failure of therapy at premortem diagnosed pulmonary thromboembolism
}

\author{
Aysun Yakar ${ }^{1}$, Fatih Yakar², Sibel Çağlar Atacan ${ }^{1}$, Muhlis Yıldız', İbrahim Üzün ${ }^{1}$
}

${ }^{1}$ Council of Forensic Medicine, Ministry of Justice, Istanbul, Turkey.

${ }^{2}$ Department of Pulmonary Medicine, Bezmialem Vakif University Medical Faculty, Istanbul, Turkey.
Correspondence: Dr. Aysun Yakar, Council of Forensic Medicine, Ministry of Justice, Istanbul, Turkey.

E-mail: draysunakdeniz@yahoo.com

\section{A B S T R A C T}

Objective: We analyzed patients diagnosed premortem with pulmonary thromboembolism (PTE), focusing on causes of failure of therapy together with clinical characteristics.

Methods: This was a retrospective study of 25 cases. We classified PTE as massive, submassive and nonmassive.

Results: Of the 25 cases, 76\% (19/25) had two or more risk factors for PTE. The point of origin of PE could be determined in only $44 \%$ of 25 cases. In $20 \%(5 / 25)$ of cases diagnosed with PTE a maximum 3 months prior to death, the main cause of death was unknown. Of 10/20 (50\%) massive and $8 / 20$ (40\%) submassive PTE cases, 5 and 3 underwent thrombolysis therapy, respectively. Both of 2/20 (10\%) non-massive PTE cases underwent $\mathrm{LMWH}$; in 1 of these cases, autopsy showed thrombus of a large pulmonary artery at 25 days after therapy. Submassive PTE cases were older and had a greater number of risk factors than did massive PTE cases. The point of origin of PTE was a right heart thrombus in six (24\%) cases. Surgeries were performed in $3 / 6(50 \%)$ cases with right atrial thrombus. The survival duration after therapy in those who underwent surgery was longer than those who received thrombolysis and LMWH therapies.

Conclusion: Physicians may provide appropriate information to patients who have certain risk factors (trauma, surgery, pregnancy, etc.) to predict acute PE at a preventable stage. If risk factors are present, or if new risk factors for PTE are identified, patients should be followed up carefully due to the risk of thrombosis progression.

Key words:Causes, failure of treatment, PTE, clinical features, fatal PTE

\section{INTRODUCTION}

Venous thromboembolism (VTE) is the third most frequent cardiovascular disease. The epidemiology of $\mathrm{PE}$ is difficult to determine because it may remain asymptomatic, or its diagnosis may be an incidental finding; in some cases, the first presentation of pulmonary embolism (PE) is sudden death. In patients with high or intermediate clinical probability for PE, parenteral anticoagulation should be initiated whilst awaiting the results of diagnostic tests [1].

Therapies such as thrombolysis $[2,3]$ or surgical embolectomy [4-6] are presumed to reduce mortality. Both methods, either alone or in combination, have been performed even in ongoing cardiopulmonary resuscitation situations.

In this retrospective study, we analyzed patients diagnosed with PE premortem, focusing on the causes of failure of therapy and the clinical characteristics of the patients.

\section{METHODS}

Forensic files stored in Forensic Science Institution in Istanbul, Turkey, from January 2010 to December 2014, were screened. Cases diagnosed with pulmonary thromboembolism (PTE) premortem were included in this study. 
The following data were collected: demographic information, medical history (including major diseases, recent surgery or trauma, and ongoing illness), signs and symptoms before death, and point of origin of $\mathrm{PE}$.

We classified PTE as massive when hypotension, shock, acute cardiopulmonary arrest and acute right ventricle dysfunction were present; sub-massive when arterial blood pressure was normal and right ventricle function had failed or the embolization involved less than two lobar arteries but at least one pulmonary segment or its equivalent; and non-massive when normal blood arterial pressure and right ventricle function were normal and the embolization involved segmental or bronchial arteries.

Deaths that fulfilled the following criteria were considered to involve PE:

a) Death was associated with acute cardiopulmonary arrest or rapidly progressive respiratory failure, and pulmonary embolism was found in the proximal pulmonary arteries by thorax computed tomography (CT), echocardiography (ECO) and/or autopsy. PE was the main cause of death.

b) The patient had an underlying disease and pulmonary embolism was found in the proximal-middle pulmonary arteries or multiple thromboemboli involving more than $50 \%$ of the pulmonary arterial bed were detected by thorax CT. PE was a contributing cause of death.

c) Pulmonary embolism was found in the segmentalsubsegmental, distal pulmonary arteries upon thorax CT and/ or the clinical data indicated that the patient would have died of his or her underlying disease. In this group, the cause of certain death was considered to be unknown.

\section{RESULTS}

Of the 25 cases with PE, 13 (52\%) were males and 12 (48\%) were females. The age range was $17-80$ years. Of the cases, $68 \%$ $(17 / 25)$ were older than 40 years, and $32 \%$ cases $(8 / 25)$ were less than 40 years of age.

PE was the main cause of death in $64 \%(16 / 25)$ of the cases. In $16 \%(4 / 25)$ of the cases, PE was a contributing factor of death. Diseases that contributed to death were coronary heart diseases, CVE (cerebrovascular event), abundant hemoptysis, pulmonary hypertension, and cardiac global dysfunction. In 20\% (5/25) of cases diagnosed with PE a maximum 3 months prior to death, the main cause of death was unknown. Patients with no definitive diagnosis had intracranial contusion after trauma, CVE, multiple metabolic diseases, and sepsis.

Diagnosis of PTE was established by only CT scan in 44\% $(11 / 25)$ of cases, by only echocardiography in $20 \%(5 / 25)$, by ventilation-perfusion lung scan in $4 \%(1 / 25)$, and by autopsy in $20 \%(5 / 25)$ of cases, in addition to imaging techniques.

Of the cases, $76 \%(19 / 25)$ had two or more risk factors and $52 \%(13 / 25)$ had three or more risk factors for PTE. Of the 25 cases, 10 (40\%) suffered multiple traumas and 4 (16\%) underwent surgery.

The point of origin of PE could be determined in only $44 \%$ of 25 cases (Table 1 ).

Table 1. Point of origin of pulmonary embolism: 11 of 25 cases.

Superior vena cava $9.09 \%$ (1)

Deep vein thrombus $36.6 \%$ (4)

Right atrium $54.54 \%$ (6)

Of 10/20 (50\%) massive PTE cases, 5 underwent thrombolysis therapy. In comparison, of 8/20 (40\%) submassive PTE cases, 3 received thrombolysis therapy. Both of $2 / 20(10 \%)$ non-massive PTE cases underwent LMWH; in 1 of these cases, autopsy showed thrombus of a large pulmonary artery at 25 days after therapy. Surgeries (embolectomy and/or pulmonary thromboendarterectomy) were performed in $3 / 20$ (15\%) cases with right atrial thrombus (Table 2).

Of five massive PTE cases who underwent thrombolysis therapy, four received r-TPA (recombinant tissue plasminogen activator), in the remaining case, the type of thrombolysis therapy was not described. Of these five cases, three had potential risk factors (trauma and pregnancy) for PTE, and four cases were $<40$ years of age. All of the five (100\%) massive PTE cases experienced hypovolemic shock; all died within 1 day.

Six cases who had submassive PTE and did not undergo surgery were $>40$ years of age. Five of these cases had three or more risk factors and one had two risk factors. Of the six cases, three suffered multiple traumas and two had metabolic disorders. Therapies administered were thrombolysis in three cases and LMWH in three cases. The majority of these cases died within 3 days.

All of the three surgery cases had right atrial thrombus. PTE was massive in one case and submassive in two cases. One of the cases underwent surgery 6 days after heparin infusion.

Of the six cases with right atrial thrombus, two had mobile thrombus. In the remaining four cases, whether or not the thrombus was mobile was not described. Of these six cases, two were pregnant, one had trauma, one had coronary artery bypass grafting, and one case had hypertension. Of six cases, three cases were $<40$ years of age. Therapy involved thrombolysis in two cases, LMWH in one case and surgery in three cases. The survival duration after therapy in those who underwent surgery was longer than those who received other therapies. Moreover, cases in which surgery was performed following heparin therapy survived for longer than did the other cases (Table 3 ).

Of the five cases in which an autopsy was performed, Behçet's disease (BD) PE, and DVT were diagnosed 3 months prior to death in one case. This individual had been administered colchicine and warfarin. Autopsy showed thrombosis in aneurysm of a pulmonary artery and deep vein of lower extremity. Of these 
five cases, one was admitted to hospital due to cellulitis. CT scan detected subsegmental thrombus, and the patient underwent LMWH for the duration of hospitalization. The patient died 1 month after initiation of LMWH therapy; the autopsy finding was thrombosis of the large pulmonary arteries. One of the five cases was pregnant and had developed deep vein thrombosis 3 months prior to death, for which subcutaneous enoxaparin was administered. In this case, autopsy showed pulmonary and femoral arterial and venous thromboses.

Table 2. Survival duration after therapy for PTE.

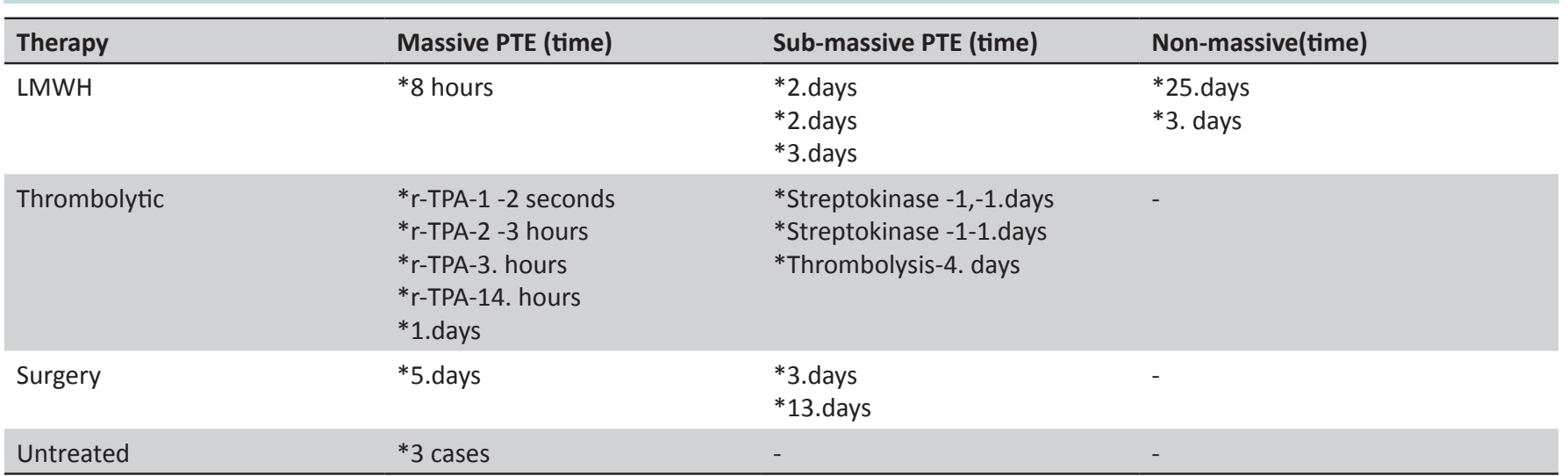

Table 3. Patients with right atrial thrombus; six cases.

\begin{tabular}{lllll}
\hline PTE & Age & Risk factors & Therapy & Survival duration (days) \\
\hline Massive & 20 & None & r-TPA & 1 \\
Massive & 24 & Pregnancy & LMWH & 1 \\
Massive & 24 & Pregnancy & Surgery & 5 \\
Sub-massive & 49 & Trauma & Streptokinase & 1 \\
Sub-massive & 76 & Bypass surgery & Surgery & 3 \\
Sub-massive & 62 & Hypertension & Surgery after heparin at 6 days & 13 \\
\hline
\end{tabular}

\section{DISCUSSION}

Anticoagulation therapy in patients with acute $\mathrm{PE}$ is recommended to prevent both early death and recurrent venous thromboembolism. Thrombolytic drugs have been shown to be more effective in terms of reducing the thrombotic burden than systemic anticoagulants alone. Accelerated regimens administered over a 2 -h period are preferable to prolonged infusions of firstgeneration thrombolytic agents over $12-24 \mathrm{~h}[1,7,8]$. In this study, of five massive PTE cases who received thrombolysis therapy, four underwent r-TPA. All five died within 1 day. The greatest benefit is observed when thrombolysis treatment is initiated within $48 \mathrm{~h}$ of symptom onset, but thrombolysis can still be useful in patients who have had symptoms for 6-14 days [1]. A fatal outcome may indicate that these cases attended hospital too late. On the other hand, a favorable outcome of thrombolytic treatment in hemodynamic unstable patients has been reported $[2,3]$. In this study, the majority of cases were of a younger age, so the determinant of mortality may have been be embolic burden rather than age, because older age is frequently associated with cardiopulmonary disease.

One of these cases was pregnant and developed deep vein thrombosis 3 months before death; this patient was administered subcutaneous enoxaparin. In this case, autopsy showed pulmonary and femoral arterial and vein thromboses. Arterial thromboses are thought to give rise to arterial complications of Behçet's disease [9]. On the other hand, because of increased renal excretion, the half-life of low-molecular-weight heparin is decreased in pregnancy [10]; therefore, a twice daily, weight-based regimen is recommended. In this case, defective treatment and follow up might have been major determinants of mortality.

Echocardiography was available in 40\% (4/10) of cases of massive PTE. Bedside diagnostic methods (echocardiography and Doppler ultrasonography) are readily available at all hospitals. Therefore, in critically ill patients, a diagnosis of PTE can be confirmed by echocardiography.

In the absence of hemodynamic compromise at presentation, the clinical benefits of thrombolysis are controversial [1, 7]. In 
the present study, six cases had submassive PTE except cases with right atrial thrombi, who underwent surgery. Administered therapies were thrombolysis and LMWH. Two studies $[4,5]$ have reported that thrombolysis in conjunction with heparin, rather than heparin alone, improves the clinical course of stable patients who have acute submassive pulmonary embolism, and thrombolysis alone had a lower composite end point of death/ recurrence. In this study, first-generation thrombolytic agents were administered in three cases of submassive PTE despite a higher risk of bleeding complications of thrombolysis with increasing age and in the presence of comorbidities. Therefore, there may have been a relationship between clot size and age and patient outcome in these cases.

Surgical embolectomy may be a good choice as it is associated with a better in-hospital course in patients with massive PE who have not responded to thrombolysis [6]. In the other cases in which LMWH was administered, thrombolysis or heparin infusion instead of LMWH could be appropriate. These submassive PTE cases were older and had greater numbers of risk factors than did the massive PTE cases. In this group, the presence of a greater number of potential risk factors and older age may be important for making treatment decisions, even in hemodynamically stable patients.

Right-sided heart thrombi (RSHT) are uncommon and often fatal [11]. Two different mechanisms seem to be involved in the formation of right-sided heart clots formed in situ (mural thrombi) versus those formed by embolization from the leg veins [12]. In situ or mural thrombi are immobile, while a thrombus in transit is highly mobile $[13,14]$. Mural RA thrombi are seen in various conditions, such as cardiomyopathies, post-atrial septal defect repair, post coronary artery bypass grafting, patients with permanent pacemaker leads or hyperalimentation catheters or ventriculoatrial shunts in situ [15].

Of the six cases with RSHT, two had mobile thrombus; in the remaining cases, whether the thrombus was mobile was not described. Of these six cases, two were pregnant, one had trauma and one case had undergone coronary artery bypass grafting. PTE may be seen in up to $98 \%$ of cases of right atrial thromboembolism [16]. In this study, all of the six cases had pulmonary embolism. The management of RSHT is problematic and the optimal therapy remains unknown. The survival rate was lower in untreated patients with pulmonary emboli than in all other groups, including untreated patients without pulmonary emboli, and the efficacy of therapy in patients with pulmonary emboli was lower than in patients without pulmonary emboli [12]. Some authors propose intravenous thrombolysis [17-19] because it is an effective, readily available therapy and can dissolve clots in several locations simultaneously; however, it has a disadvantage in terms of migration of fragments following clot lysis $[17,18]$. In such patients, surgical embolectomy remains the treatment of choice. It may be repaired a PFO at that surgery time .In the International Cooperative Pulmonary Embolism Registry [19], the mortality rate at 14 days following embolectomy was higher than for other therapies. On the other hand, death can occur in patients awaiting surgery.
Heparin therapy has been suggested in clinically stable patients [12]. However, the onset of action of heparin is too slow even in hemodynamically stable patients, so pulmonary and systemic circulation may worsen [17]. In this study, survival duration following surgery was longer than following the other types of therapy, and survival following surgery after heparin therapy was further prolonged. Of the three surgery groups with RSHT, two had submassive pulmonary thromboembolism. In cases that underwent surgery following heparin therapy, application of thrombolysis might have resulted in a favorable outcome. On the other hand, all cases of massive PTE were younger than submassive PTE cases. One case received thrombolysis, and one received LMWH to systemic thrombolytic therapy or in those patients in whom the use of anticoagulants and thrombolytics is contraindicated [8]. In this study, there was insufficient time for surgery in patients who had been administered anticoagulation therapy. Such surgeries would require interventional equipment, sufficient time and more-experienced interventionalists.

In the present study, autopsies were diagnostic in five cases. Of these, one case had BD; in addition, PE and DVT were diagnosed 3 months prior to death. He was admitted to hospital with fever and left chest pain 2 months before death; thorax CT was not performed. His INR (International Normalized Ratio) control was 4 and warfarin was stopped at 1 day prior to death. The patient exhibited abundant hemoptysis and hypovolemic shock at the time of death. Autopsy showed thrombosis in aneurysm of pulmonary arterial and deep vein of lower extremity. Both veins and arteries of all sizes can be involved in BD [20]. Vascular involvement has been considered to result from systemic vasculitis [21]. Although rare, $\mathrm{BD}$ complicated by right ventricular thrombosis and/or pulmonary embolism has been reported [22,23]. Patients commonly present to the hospital with fever, rash, cough, dyspnea, chest pain, weight loss, and hemoptysis. Fever is more common when the pulmonary artery is involved [24-26]. In this case, the initial symptoms ( 2 months before death) were fever and left chest pain. So this condition should be kept in mind in similar cases. Patients with BD diagnosed with PE should be monitored closely to follow up the size of the thrombus. Treatment of thrombus with warfarin therapy in addition to oral steroids and immunosuppressive drugs is related to disappearance of the thrombus in most patients [27-29]. Warfarin therapy alone was ineffective according to the autopsy findings in this case. Thus, obtaining a thorough medical history, especially in patients diagnosed with PTE, is important for treatment and management.

Of five cases in which autopsy was performed, one case was admitted to hospital for cellulitis. A CT scan detected subsegmental thrombus, and the patient received LMWH for the duration of hospitalization. This patient died 1 month following initiation of LMWH therapy. The finding at autopsy was thrombus of the large pulmonary arteries. In hospital, the potential risk factors for PTE in this case were immobilization and catheter use. Withholding treatment for subsegmental PTE appears to be safe, provided that the pulmonary respiratory reserve is good, there is no evidence of DVT on serial testing, the major risk factor for 
PTE is transient and no longer present, and there is no history of central venous catheterization or atrial fibrillation [30]. Thus, in this case, symptoms should have been monitored frequently and imaging techniques should have been performed serially, because of the continual presence of multiple risk factors.

\section{CONCLUSIONS}

Correct diagnosis and treatment planning should be determined based on the symptoms, physical examination, imaging techniques (computed tomography, Doppler ultrasonography, and echocardiography), and a medical history. Physicians should provide appropriate information to patients with certain risk factors (trauma, surgery, pregnancy etc.) to predict acute PE at a preventable stage. Early diagnosis may result in a good outcome. Patients with BD may develop pulmonary embolism and right ventricular thrombus as part of systemic vascular complications. It is possible that the combination of oral steroid, cyclophosphamide and warfarin may be effective for treatment of pulmonary thrombus in patients with Behçet's disease. If risk factors for PE continue to be present, patients should undergo careful follow up because of the risk of progression of thrombosis. In this study, several subgroups comprised relatively few patients, so a greater number of patients is required to determine the reasons for failure of PTE treatment.

Declaration of Conflicting Interests: The authors declare that they have no conflict of interest.

Financial Disclosure: No financial support was received.

\section{REFERENCES}

1. Konstantinides SV, Torbicki A, Agnelli G, et al. 2014 ESC Guidelines on the diagnosis and management of acute pulmonary embolism. Eur Heart J. 2014;35:3033-80.

2. Kürkciyan I, Meron H, Sterz F, et al. Pulmonary embolism as cause of cardiac arrest. Arch Intern Med. 2000;160:1529-35.

3. Böttiger BW, Böhner H., Bach A, et al. Bolus injection of thrombolytic agents during cardiopulmonary resuscitation for massive pulmonary embolism. Resuscitation. 1994;28:45-54.

4. Konstantinides SV, Geibel A, Heusel G, et al. Heparın plus Alteplase compared with heparin alone in patients with submassive pulmonary embolism. N Engl J Med. 2002;347:1143-50.

5. Agnelli G, Becattini C, Kirschstein T. Thrombolysis vs. heparin in the treatment of pulmonary embolism. Clinical outcome based meta-analysis. Arch Intern Med. 2002;162:2537-41.

6. Meneveau N, Séronde MF, Blonde MC, et al. Management of unsuccessful thrombolysis in acute massive pulmonary embolism. Chest. 2006;129:1043-50.

7. Wood K.E. Major Pulmonary Embolism. Crit Care Clin. 2011;27:885906.

8. Saad N. Aggressive Management of Pulmonary Embolism. Semin Intervent Radiol. 2012;29:52-6.
9. Bensaid Y, Ameur A, Kabiri H, et al.: Arterial complications of Behcet's disease. Report of 13 cases. J Mal Vasc. 1997;22:24-8.

10. Marik PE, Plante LA. Venous thromboembolic disease and pregnancy. $\mathrm{N}$ Engl J Med.

11. Wartman WB, Hellerstein HK, The incidence of heart disease in 2000 consecutive autopsies. Ann Intern Med. 1948;28:41-65.

12. Kinney EL, Wright RJ. Efficacy of treatment of patients with echocardiographically detected right-sided heart thrombi-a meta analysis. Curriculum Cardiol.1989;9:12-9.

13. European Working Group on Echocardiography and pulmonary embolism. Ann Intern Med. 1977;7:720-1.

14. Farfel Z, Shechter M, Vered Z. et al. Review of echocardiographically diagnosed right heart entrapment of pulmonary emboli in transit with emphasis on management. Am Heart J. 1987;113:171-8.

15. Panidis IP, Kotler MN, Mintz GS, et al. Clinical and echocardiographic features of right atrial masses. Am Heart L. 1984;107:745-58.

16. Rose PS., Punjabi NM., Pearse DB. Treatment of right heart thromboemboli. Chest, 2002;121:806-814.

17. Pierre-Justin G, Pierard L.A. Management of mobile right hearts thrombi: A prospective series. Int J Cardiol. 2005;99:381-8.

18. Mohan B, Chhabra ST, Gulati A, et al. Clinical and echocardiographic diagnosis, follow up and management of right sided cardiac thrombi. Indian Heart J. 2013;65:529-35.

19. Torbicki A, Galie N, Covezzoli A, et al. Right Heart Thrombi in Pulmonary Embolism. Results from the International Cooperative Pulmonary Embolism Registry. J Am Coll Cardiol. 2003;41:2245-51.

20. Sahin M, Arslan C, Tunc SE, et al. A fatal case of Behçet's disease with rare complications. Saudi Med J 2006;27:1754-7.

21. Kale A, Aky1ldız L, Akdeniz N, et al. Pregnancy complicated by superior vena cava thrombosis and pulmonary embolism in a patient with Behçet's disease and the use of heparin for treatment. Saudi Med J. 2006;27:95-7.

22. Saghil G, Doghmil N. Intracardiac thrombosis in Behçet's disease: a rare complication. Pan African Med J. 2013;15:91-3.

23. Marc K., Iraqui G., Jniene A. et al. [Intracardiac thrombus and pulmonary artery aneurysm in Behçet's disease]. Rev Mal Respir. 2008;25:69-72.

24. Xing W, Swaminathan G, Appadorai DR, et al. Rare case of Behçet's disease presenting with pyrexia of unknown origin, pulmonary embolism, and right ventricular thrombus. Int J Angiol. 2013;22:193-8.

25. Chang JE, Lee YH, Lee J. Multiple cardiovascular complications in a patient with Behcet's disease. Korean J Intern Med. 2008;23:100-2.

26. San Luis Miranda R., Lázaro Castillo JL., Enciso Gómez R. et al. [Right ventricular thrombus and pulmonary artery aneurysms in Behçet's disease. Report of one case]. Arch Cardiol Mex. 2007;77:130-6.

26. Desbois AC1, Wechsler B, Resche-Rigon M, et al. Immunosuppressants reduce venous thrombosis relapse in Behçet's disease. Arthritis Rheum. 2012;64:2753-60.

27. Hammami R., Abid L., Frikha F. et al. Intracardiac thrombus in a young man: don't forget Behçet's disease. Intern Med. 2012;51:1865-7.

28. Dogan SM, Birdane A, Korkmaz C, et al. Right ventricular thrombus with Behçet's syndrome: successful treatment with warfarin and immunosuppressive agents. Tex Heart Inst J. 2007;34:360-2.

29. Stein PD, Goodman LR, Hull RD, et al. Diagnosis and management of isolated subsegmental pulmonary embolism; review and assessment of the options. Clin Appl Thromb Hemost. 2012;18:20-6. 